\title{
Vertebral column decancellation for the management of sharp angular spinal deformity
}

\author{
Yan Wang • Lawrence G. Lenke
}

Received: 13 July 2010/Revised: 19 February 2011/Accepted: 10 March 2011/Published online: 19 March 2011

(C) The Author(s) 2011. This article is published with open access at Springerlink.com

\begin{abstract}
The management goal of sharp angular spinal deformity is to realign the spinal deformity and safely decompress the neurological elements. However, some shortcomings related to current osteotomy treatment for these deformities are still evident. We have developed a new spinal osteotomy technique-vertebral column decancellation (VCD), including multilevel vertebral decancellation, removal of residual disc, osteoclasis of the concave cortex, compression of the convex cortex accompanied by posterior instrumentation with pedicle screws, with the expectation to decrease surgical-related complications. From January 2004 to March 2007, 45 patients (27 males/18 females) with severe sharp angular spinal deformities at our institution underwent VCD. The diagnoses included 29 congenital kyphoscoliosis and 16 Pott's deformity. Preoperative and postoperative radiographic evaluation was performed. Intraoperative, postoperative and general complications were noted. For a kyphosis deformity, an average of 2.2 vertebrae was decancellated (range, 2-4 vertebrae). The mean preoperative kyphosis was $+98.6^{\circ}$ (range, $82^{\circ}-138^{\circ}$ ), and the mean kyphosis in the immediate postoperative period was $+16.4^{\circ}\left(\right.$ range, $\left.4^{\circ}-30^{\circ}\right)$ with an average postoperative
\end{abstract}

This manuscript is not sponsored directly or indirectly by a pharmaceutical company, equipment manufacturer, public relations firm, or any other commercial entity.

Y. Wang $(\bowtie)$

Department of Orthopaedics, Chinese PLA General Hospital, 28 Fuxing Road, 100853 Beijing, People's Republic of China e-mail: 301wangyan@sina.com

L. G. Lenke

Department of Orthopaedic Surgery, Shriners Hospital for Children, Washington University School of Medicine, St. Louis, USA correction of $+82.2^{\circ}\left(\right.$ range, $61^{\circ}-124^{\circ}$ ). For a kyphoscoliosis deformity, the correction rate was $64 \%$ in the coronal plane (from $83.4^{\circ}-30.0^{\circ}$ ) postoperatively and $32.5^{\circ}(61 \%$ correction) at 2 years' follow-up. In the sagittal plane, the average preoperative curve of $88.5^{\circ}$ was corrected to $28.6^{\circ}$ immediately after surgery and to $31.0^{\circ}$ at 2 years' followup. All patients had solid fusion at latest follow-up. Complications were encountered in eight patients (17.8\%), including CSF leak $(n=1)$, deep wound infection $(n=1)$, epidural hematoma $(n=1)$, transient neurological deficit $(n=4)$, and complete paralysis $(n=1)$. The results of this study show that single-stage posterior VCD is an effective option to manage severe sharp angular spinal deformities.

Keywords Posterior approach .

Vertebral column decancellation (VCD) .

Kyphosis · Kyphoscoliosis · Osteotomy

\section{Introduction}

The presence of a kyphotic or kyphoscoliotic deformity in various pathologic conditions on radiographs does not necessarily imply the need for treatment, either operative or nonoperative. However, patients with sharp angular spinal deformities may have neurologic symptoms, such as late-onset paraparesis or even paraplegia, low back pain, and significant cosmetic problems, and psychological implications. Surgery may be the appropriate option for the treatment of these patients.

Several different spinal osteotomies are available for treating rigid spinal deformities, including an anterior opening wedge osteotomy (OWO), e.g. Smith-Petersen osteotomy (SPO), closing wedge osteotomy (CWO), e.g. pedicle subtraction osteotomy (PSO) $[4,16]$, a 

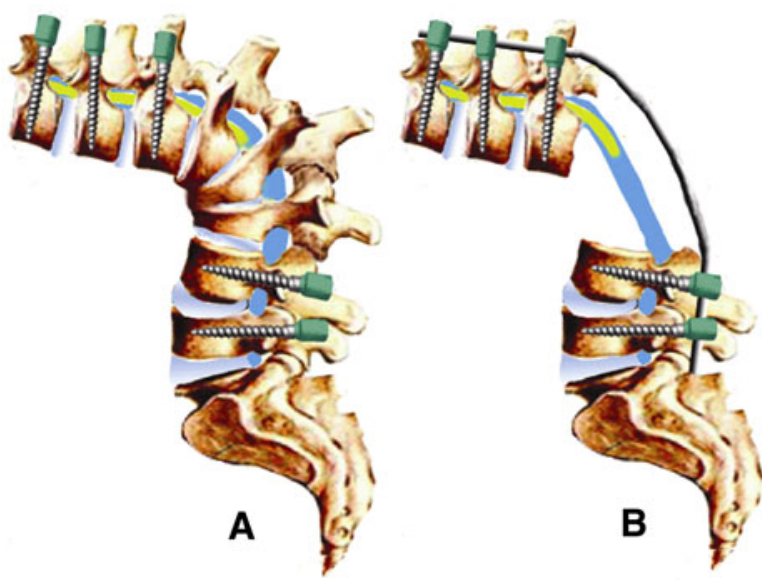

Fig. 1 VCR diagrams. a Pedicle screws were inserted before the osteotomy was performed. b A temporary rod was placed to bridge the anterior resection before beginning the posterior osteotomies to provide stability and reduce the chances of sudden spinal translation.

closing-opening wedge osteotomy, and a vertebral column resection (VCR) [3, 7]. The SPO technique includes resection of the posterior column and opening of the anterior column through a mobile disc space. The PSO technique applies resection of the posterior column and both pedicles, and wedging of the vertebral body, with the hinge located at the anterior cortex of the vertebral body [1]. The VCR technique involves complete resection of one or more vertebral segments through a combined anterior and posterior approach [2] or via a posterior-only approach $[11,14,15]$, with the anterior column reconstructed with metal mesh filled with morselized bone graft (Fig. 1).

Theoretically, a closing-opening technique, such as the VCR, may be the best option for the management of severe angular spinal deformity, because it can provide adequate canal decompression and excellent correction of the sagittal and coronal planes while shortening the length of the spinal column. However, limitations of the VCR are evident and include spinal column instability, greater blood loss, extensive manipulation performed in spinal cord territory, and greater risk of neurologic deficit [3].

Do we have to resect the deformed vertebrae described in the VCR technique like the management of spinal tumor? In fact, the corrective spinal osteotomy may be considered to realign the patient's deformed spine and decompress the neural elements. Therefore, it is a negative answer to the question. We have developed a new spinal osteotomy-vertebral column decancellation (VCD), including multilevel vertebral decancellation, removal of residual disc, osteoclasis of the concave cortex, compression of the convex cortex accompanied by posterior instrument with pedicle screws, with the expectation to

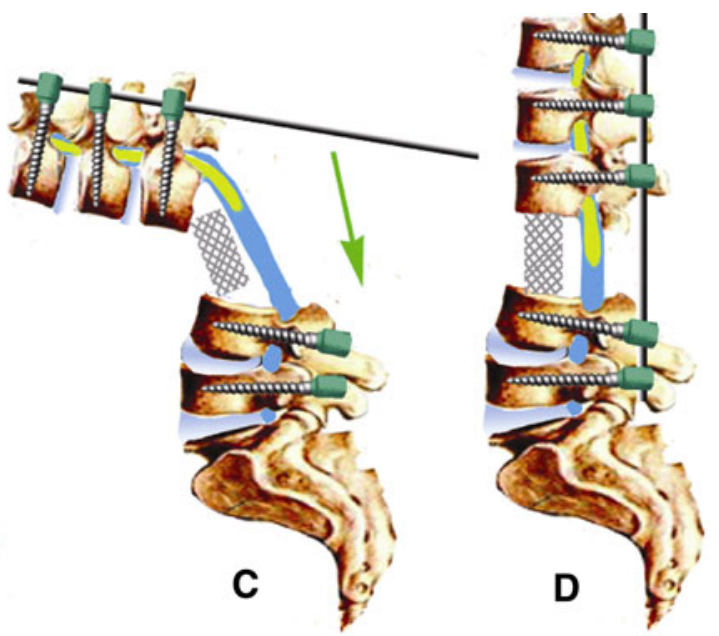

c The anterior column was reconstructed with metal mesh filled with cracked bone after the osteotomy; $\mathbf{d}$ Realignment was achieved when the posterior column was closed

decrease surgery-related complications. The goal of this study was to report the new osteotomy technique of VCD (Fig. 2) for the management of sharp angular spinal deformities.

\section{Materials and methods}

45 patients (27 males, 18 females) with severe sharp angular spinal deformities underwent VCD at our institution from January 2004 to March 2007. Diagnoses included 29 patients with congenital kyphoscoliosis and 16 with Pott's kyphotic deformity. The main complaints from patients included neurological deficits of the lower extremities, cosmetic issues, fatigue or low back pain, being unable to lie down in a dorsal position, and the inability to stand erect despite compensatory hip extension, knee flexion, etc. One patient with Pott's deformity suffered digestive system dysfunction and painful costo-pelvic impingement. One patient was Grade $\mathrm{C}$ and three patients were Grade D with regard to neurologic deficit according to the ASIA Impairment Scale [5].

There are two main curve types in sharp angular spinal deformities: kyphosis and kyphoscoliosis. In patients with Pott's disease, the curve type is usually a kyphosis deformity. In general, the younger the patients were infected with tuberculosis, the better global sagittal balance the spine can achieve, because the upper and lower regions of the deformity can compensate for the kyphosis during spinal growth. These spinal alignment changes also influence the selection of instrumentation levels. 


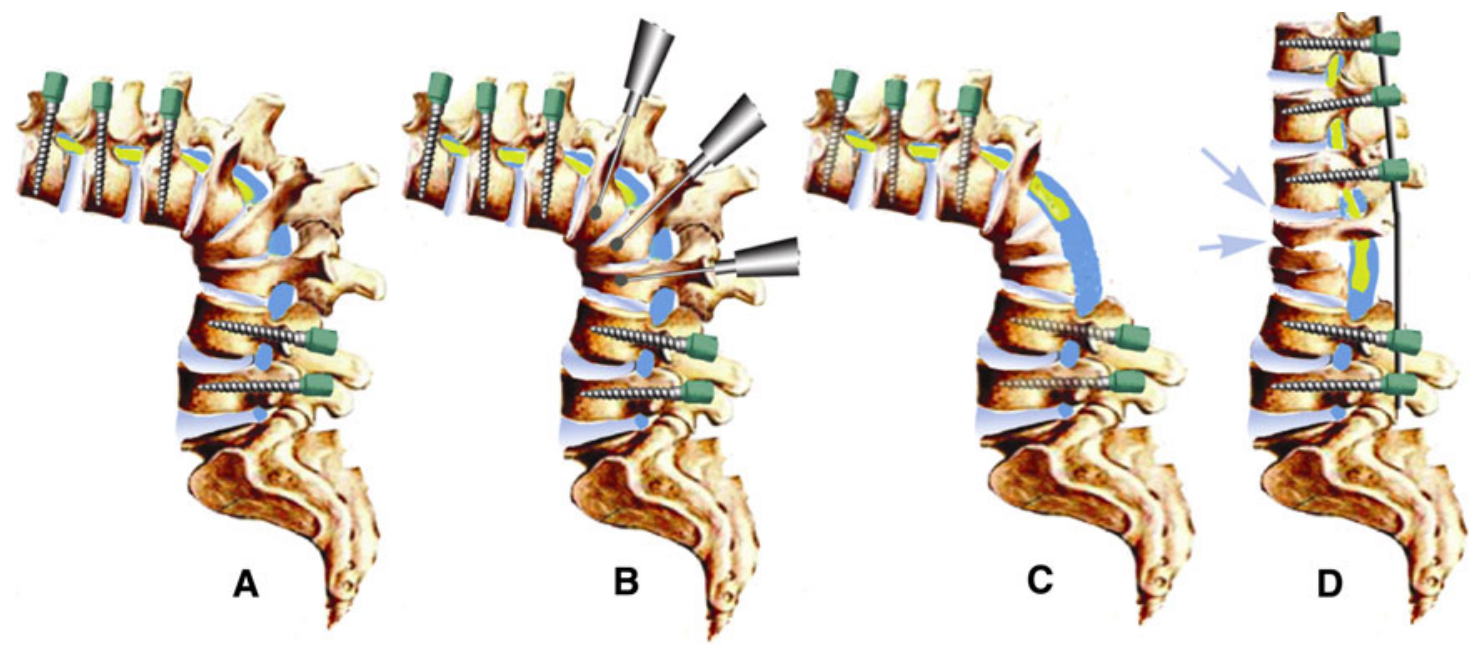

Fig. 2 VCD diagrams. a Pedicle screws were inserted before the osteotomy was performed. b A high-speed drill was used to decancellate the deformed vertebrae. c Many posterior elements were removed along with the residual disc. d Postoperative lateral view shows that correction is achieved by elongating and opening (arrow) the anterior column and shortening the posterior column, and the residual bone takes the place of metal mesh described in the VCR technique, serving as a "bony cage"
The surgical plan, including the resection site, instrumentation levels and degree of correction was determined based on clinical radiographs, CT scans, 3D reconstruction images, and MRI exams. All surgeries were monitored by intraoperative somatosensory-evoked potentials. All patients were evaluated by X-ray before and immediately after surgery, then 3, 6 and 12 months postoperatively and yearly thereafter. Intraoperative, postoperative and general complications were noted.

\section{Operative technique}

In the kyphosis correction technique, a standard skin incision was made in the midline, and a subperiosteal dissection was performed to expose the bony structures of the posterior elements. All pedicle screws were inserted via a free hand pedicle screw placement technique [9]. The VCD begun with the probe of the pedicle of deformed vertebral body to be removed. C-arm fluoroscopy confirmed the appropriate plane for the osteotomies. Then, a high-speed drill was used to enlarge the pedicle hole both cephalad and caudad until the corresponding walls were penetrated. An angular forceps or curette was used to remove the residual upper and lower cartilaginous endplates of the resected vertebra and intervertebral discs; then, a Kerrison rongeur or drill was used to thin the anterior and lateral walls of the vertebral body, which collapsed under pressure laterally to expose the posterior walls further. The posterior wall was resected with angular forceps. Most of the time, it was unnecessary to remove the cancellous bone entirely, and the residual bone was used to reconstruct a "bony cage" to take place of metal mesh as described in VCR techniques. After the vertebral bodies were decancellated, the posterior elements, including the spinous processes, laminae, facet joints, and transverse processes were removed. Osteoclasis of the anterior cortex of vertebral body was achieved by gentle manual extension of the upper body and the lumbar spine to close posterior wedge osteotomies, creating an anterior mono-segmental intervertebral opening wedge or osteoclasis of the anterior cortex with elongation of the anterior column. The hinge of the correction was located at the border of the anterior and medial column. If the correction was not adequate, a retroposition of the hinge would be performed. If the cavity was too large to contact the two osteotomy planes, autogenous bone was then placed at the osteotomy site gap with the expectation of better bony fusion and better stability. After confirmation of correction via fluoroscopy, final internal fixation was applied (Fig. 3).

In the kyphoscoliosis technique, the basic techniques were similar to those in the kyphosis correction. The difference was that the consideration of correction was also about the coronal plane rather than sagittal plane only. Decancellation was performed mainly on the convex side in the same fashion. The spine on the convexity was then closed, while segmental compression was achieved. After internal fixation, autograft bone chips were implanted into the residual intervertebral spaces. In some cases, when multilevel posterior structures were removed, the posterior gap was unable to be closed by compression with the rods only, so we harvested iliac bone, in the form of matchsticks, and implanted them into the posterior space and/or placed them over the transverse processes. 

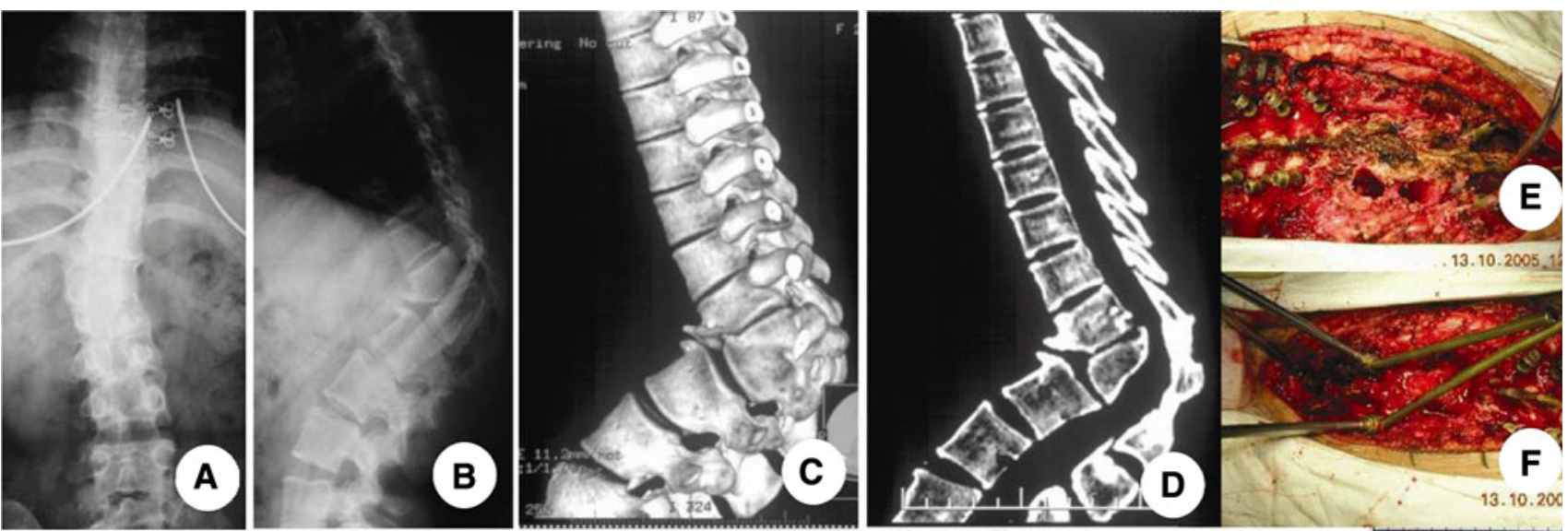

$13.10 \cdot 2005.13$
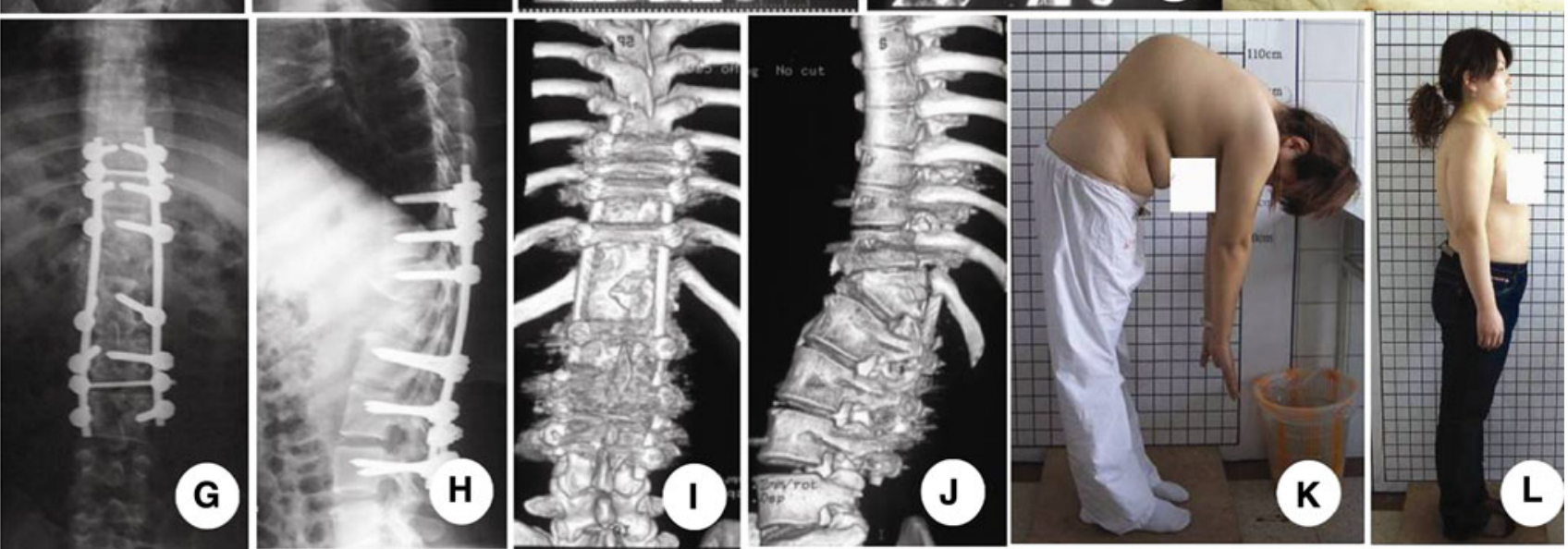

Fig. 3 A 24-year-old patient with Pott's disease. The patient's main complaints were low back pain and cosmetic issues. a-d Preoperative $\mathrm{X}$-ray and CT scan reconstruction showed a sharp angle in thoracolumbar spine. $\mathbf{e}, \mathbf{f}$ Intraoperative picture of a vertebral column decancellation (VCD) was taken at T12 and L1. The VCD was begun with the probe of the pedicle of deformed vertebral body. A highspeed drill was used to enlarge the two pedicle holes. The residual

upper and lower cartilaginous endplates and discs, posterior walls of the vertebral body and posterior elements were removed carefully, followed by cantilever technique and pedicle instrument; $\mathbf{g}, \mathbf{j} 3$ years' follow-up X-ray and CT scan show a solid fusion between T12 and L1. k, I Preoperative and postoperative clinical pictures show the improved cosmesis

During the exposure and bone resection, care should be taken to perform meticulous hemostasis. It is essential to use bipolar cautery to control bleeding in the soft tissues and to coagulate epidural vessels as needed. Absorbable hemostatic gauze and gelatin sponge were packed at the wound sites as needed. Three methods were used to replenish blood loss: (1) Cell saver, which re-transfuses the patient's own blood; (2) blood from the blood bank was also given to the patient when necessary; (3) and for the first $6 \mathrm{~h}$, blood from the suction drains was collected and remains fused using a postoperative cell saver.

\section{Postoperative management}

Closed suction drains were inserted at the resection site in all patients. All patients were extubated immediately after the procedure. Drains were discontinued when blood collection was $<50 \mathrm{ml}$ per $24 \mathrm{~h}$. Patients were typically allowed to ambulate within $48-72 \mathrm{~h}$ using a thoracolumbosacral

orthosis, which was worn thereafter with ambulation for 3 months.

\section{Results}

There were 27 males and 18 females, with a mean age of 28.6 years (range, 16-54 years), all with a sharp angular spinal deformity who underwent the VCD technique. A mean of 7.6 vertebral levels were instrumented and fused (range, 6-12 vertebrae). The mean duration of surgery was $276 \mathrm{~min}$ (range, 236-412 $\mathrm{min}$ ). The average intraoperative blood loss was 2,812 $\mathrm{ml}$ (range, 1,864-6,000 ml).

\section{Radiographic outcomes}

An average of 2.2 vertebrae was decancellated in each case (range, 2-4 vertebrae). For the kyphosis group, the mean preoperative kyphosis was $98.6^{\circ}$ (range, $82^{\circ}-138^{\circ}$ ), and the 
Table 1 Correction of deformity in kyphosis group

\begin{tabular}{|c|c|c|c|c|c|c|}
\hline \multirow[t]{2}{*}{ Patients: cases } & \multicolumn{4}{|c|}{ Correction results } & \multicolumn{2}{|l|}{ Operation condition } \\
\hline & Preop $\left(^{\circ}\right)$ & Postop $\left({ }^{\circ}\right)$ & Correction $\left(^{\circ}\right)$ & 2 years postop $\left(^{\circ}\right)$ & Osteotomy site & Instrumentation segments \\
\hline 1 & 90 & 30 & 65 & 32 & $\mathrm{~T} 11, \mathrm{~T} 12$ & T7-10, L1-3 \\
\hline 2 & 100 & 25 & 75 & 28 & $\mathrm{~T} 12, \mathrm{~L} 1$ & T9-11, L2-4 \\
\hline 3 & 132 & 9 & 123 & 9 & $\mathrm{~T} 12, \mathrm{~L} 1, \mathrm{~L} 2$ & T8-11, L3-4 \\
\hline 4 & 90 & 29 & 61 & 30 & T9, T10 & T6-8, T11-L1 \\
\hline 5 & 88 & 14 & 81 & 15 & $\mathrm{~T} 12, \mathrm{~L} 1$ & T9-11, L2-4 \\
\hline 6 & 102 & 30 & 72 & 36 & $\mathrm{~T} 4, \mathrm{~T} 5$ & T1-3, T6-11 \\
\hline 7 & 98 & 16 & 81 & 16 & $\mathrm{~T} 11, \mathrm{~T} 12$ & T7-10, L1-3 \\
\hline 8 & 95 & 10 & 100 & 12 & $\mathrm{~T} 11, \mathrm{~T} 12, \mathrm{~L} 1$ & $\mathrm{~T} 8-10, \mathrm{~L} 2-4$ \\
\hline 9 & 86 & 16 & 70 & 20 & $\mathrm{~T} 12, \mathrm{~L} 1$ & T9-11, L2-4 \\
\hline 10 & 138 & 14 & 124 & 15 & $\mathrm{~T} 11, \mathrm{~T} 12, \mathrm{~L} 1, \mathrm{~L} 2$ & T6-11, L3-5 \\
\hline 11 & 99 & 29 & 70 & 34 & T3-6 & $\mathrm{C} 7-\mathrm{T} 2, \mathrm{~T} 7-11$ \\
\hline 12 & 93 & 4 & 89 & 4 & $\mathrm{~T} 11, \mathrm{~T} 12, \mathrm{~L} 1$ & T8-10, L2-4 \\
\hline 13 & 85 & 6 & 79 & 8 & L1, L2 & T9-12, L3-4 \\
\hline 14 & 108 & 10 & 98 & 12 & L1, L2, L3 & T9-T12, L4-5 \\
\hline 15 & 91 & 11 & 80 & 16 & $\mathrm{~T} 11, \mathrm{~T} 12, \mathrm{~L} 1$ & T8-10, L2-4 \\
\hline 16 & 82 & 10 & 72 & 12 & L2, L3 & T9-L1, L4-5 \\
\hline Average & 98.6 & 16.4 & 83.8 & 18.7 & & \\
\hline
\end{tabular}

mean kyphosis in the immediate postoperative period was $16.4^{\circ}$ (range, $4^{\circ}-30^{\circ}$ ) with an average postoperative kyphosis correction of $82.2^{\circ}$ (range, $61^{\circ}-124^{\circ}$ ) (Table 1). For the kyphoscoliosis group, the correction rate immediately postoperative was $64 \%$ in the coronal plane (from $83.4^{\circ}$ to $30.0^{\circ}$ ), and $61 \%$ at 2 years follow-up. In the sagittal plane, the average correction was from $88.5^{\circ}$ to $28.6^{\circ}$ immediately after surgery, and $31.0^{\circ}$ at 2 years follow-up (Table 2). All patients showed evidence of a solid fusion at the last follow-up.

\section{Clinical outcomes}

The cosmetic issues of all patients have been improved obviously. All patients can lie down in a dorsal position and can stand without compensatory of hip extension. The patient with digestive system dysfunction and painful costo-pelvic impingement also has been improved. All patients complained that the low back pain was improved except one patient with severe kyphoscoliosis who complained of slight low back pain at 2 years' follow-up.

\section{Complications}

A total of eight patients (17.8\%) encountered surgical complications. One patient, whose preoperative ASIA scale is grade $\mathrm{C}$, suffered complete permanent paralysis even with attempts to improve the neurologic loss. Four patients (3 patients were Grade D and 1 was Grade E before operation) suffered transient neurological deterioration and spontaneously improved without further treatment within 3 months. One patient suffered a CSF leak secondary to a dural tear. One patient experienced an epidural hematoma, which was related to the ventral decompression. One patient developed a deep wound infection 2 months postoperatively and the infection cleared following thorough debridement. No major acute complications such as death occurred. There were no approach-related complications such as hemothorax or pleural effusion. No other complications, such as pseudarthrosis, screw misplacement or implant failure were detected during the follow-up period.

\section{Discussion}

Patients with severe rigid spinal deformity present a formidable challenge to the spine surgeon, because most of these patients need a major spinal osteotomy to achieve adequate correction. However, surgeons must answer the following critical questions when evaluating these types of patients. What patients need do and what we can do?

For sharp angular kyphotic deformity, the lever arm of the center of the gravity line with respect to the wedged vertebra may increase [17]; this change leads to the anterior elements of the spine failing under compression and the posterior elements failing under tension. This combination results in an increase in the eccentric loads, wedging, deformity, pain, and the potential for neurologic deficit 
Table 2 Correction of deformity in kyphoscoliosis group

\begin{tabular}{|c|c|c|c|c|c|c|c|c|c|}
\hline \multirow{2}{*}{$\begin{array}{l}\text { Patients: } \\
\text { cases }\end{array}$} & \multicolumn{5}{|c|}{ Coronal plane } & \multicolumn{4}{|c|}{ Sagittal plane } \\
\hline & $\begin{array}{l}\text { Preop } \\
\left({ }^{\circ}\right)\end{array}$ & $\begin{array}{l}\text { Postop } \\
\left({ }^{\circ}\right)\end{array}$ & $\begin{array}{l}\text { Correction } \\
(\%)\end{array}$ & $\begin{array}{l}2 \text { years postop } \\
\left({ }^{\circ}\right)\end{array}$ & $\begin{array}{l}2 \text { years correction } \\
(\%)\end{array}$ & $\begin{array}{l}\text { Preop } \\
\left({ }^{\circ}\right)\end{array}$ & $\begin{array}{l}\text { Postop } \\
\left({ }^{\circ}\right)\end{array}$ & $\begin{array}{l}\text { Correction } \\
\left({ }^{\circ}\right)\end{array}$ & $\begin{array}{l}2 \text { years postop } \\
\left({ }^{\circ}\right)\end{array}$ \\
\hline 1 & 78 & 24 & 69 & 26 & 67 & 78 & 31 & 47 & 33 \\
\hline 2 & 87 & 28 & 68 & 30 & 66 & 93 & 36 & 57 & 42 \\
\hline 3 & 75 & 36 & 52 & 36 & 52 & 84 & 12 & 62 & 26 \\
\hline 4 & 71 & 26 & 50 & 41 & 43 & 105 & 20 & 85 & 31 \\
\hline 5 & 81 & 31 & 63 & 32 & 61 & 82 & 30 & 52 & 32 \\
\hline 6 & 77 & 32 & 58 & 33 & 57 & 78 & 23 & 55 & 27 \\
\hline 7 & 111 & 34 & 69 & 33 & 70 & 120 & 36 & 84 & 39 \\
\hline 8 & 75 & 28 & 62 & 35 & 53 & 80 & 38 & 42 & 41 \\
\hline 9 & 75 & 32 & 57 & 33 & 57 & 79 & 30 & 49 & 28 \\
\hline 10 & 96 & 38 & 62 & 39 & 59 & 106 & 33 & 73 & 37 \\
\hline 11 & 82 & 39 & 53 & 37 & 54 & 93 & 27 & 66 & 31 \\
\hline 12 & 76 & 23 & 69 & 24 & 68 & 79 & 20 & 59 & 22 \\
\hline 13 & 71 & 32 & 54 & 37 & 49 & 99 & 22 & 77 & 28 \\
\hline 14 & 96 & 28 & 70 & 30 & 69 & 100 & 40 & 60 & 42 \\
\hline 15 & 82 & 24 & 71 & 24 & 71 & 86 & 36 & 50 & 38 \\
\hline 16 & 118 & 42 & 64 & 45 & 62 & 136 & 32 & 102 & 32 \\
\hline 17 & 84 & 30 & 65 & 30 & 65 & 88 & 24 & 64 & 28 \\
\hline 18 & 76 & 24 & 68 & 26 & 66 & 80 & 26 & 54 & 28 \\
\hline 19 & 74 & 23 & 69 & 25 & 66 & 78 & 32 & 46 & 32 \\
\hline 20 & 84 & 35 & 58 & 37 & 56 & 86 & 26 & 60 & 26 \\
\hline 21 & 83 & 26 & 69 & 30 & 64 & 80 & 24 & 56 & 28 \\
\hline 22 & 77 & 28 & 64 & 30 & 61 & 72 & 30 & 42 & 30 \\
\hline 23 & 85 & 25 & 71 & 29 & 66 & 80 & 27 & 53 & 28 \\
\hline 24 & 79 & 27 & 68 & 30 & 62 & 75 & 31 & 44 & 32 \\
\hline 25 & 84 & 36 & 57 & 40 & 52 & 85 & 28 & 57 & 30 \\
\hline 26 & 88 & 29 & 67 & 32 & 64 & 82 & 25 & 57 & 26 \\
\hline 27 & 94 & 35 & 64 & 37 & 61 & 104 & 36 & 68 & 35 \\
\hline 28 & 76 & 26 & 66 & 29 & 62 & 70 & 25 & 45 & 27 \\
\hline 29 & 83 & 29 & 65 & 33 & 60 & 88 & 28 & 60 & 30 \\
\hline Average & 83.4 & 30.0 & 64 & 32.5 & 61 & 88.5 & 28.6 & 59.5 & 31.3 \\
\hline
\end{tabular}

may develop or increase. Therefore, sufficient restoration of alignment may not only result in better fusion, but also in better spinal canal decompression. Patients with sharp angular kyphoscoliosis also face the same conditions; therefore, spinal osteotomy is a viable surgical option.

Traditionally, an SPO requires a mobile disc space anteriorly, which is uncommon with rigid sharp angular spinal deformity [19]. Though a PSO can be applied to patients with sharp angular kyphosis and anterior column fusion [3], the excessive shortening of the area may result in buckling of the dura and spinal cord, which is very dangerous, and authors have recommended limiting the correction to $30^{\circ}$ or $40^{\circ}[6,8,10]$. Recently, a single-stage posterior VCR has become increasingly popular for sharp angular spinal deformity $[2,12,13,15]$.
However, the VCR technique requires complete removal of the deformed vertebrae to allow restoration of alignment and decompression, which endangers the stability of the anterior and medial columns. Also, the anterior column needs to be reconstructed with metal mesh filled with morselized bone after the osteotomy. The limitations of these techniques undoubtedly increase surgical-related complications, such as spinal instability, in addition to greater blood loss and higher risk of neurologic deficit. These limitations were confirmed by Bradford's study [2] in which a total of 31 complications in 14 out of 24 patients who underwent a VCR for the treatment of rigid coronal deformity was reported.

We have developed a new spinal osteotomy technique with the expectation to decrease surgery-related 
complications, and have defined it as modified vertebral column resection (MVCR) before [17, 18]. In fact, this is a new type of osteotomy, and we now call this method the VCD technique to help distinguish it from previous methods.

The technique of VCD was based on the recognition that (1) decancellation of deformed vertebrae may facilitate realignment of the angular spine; (2) spine realignment may result in pain relief or relief of neurological deficits and correction of any cosmetic issues; (3) rational management of sharp angular spinal deformity can be achieved by lengthening the anterior elements, shortening the posterior elements or combination of the two. The osteoclasis of the anterior cortex of the deformed vertebrae may help to open and elongate the anterior column, which may decrease the need for shortening of the posterior column, decreasing the risk of neurological deficits; (4) residual bone of the deformed vertebrae may take the place of metal mesh described in the VCR technique, which serves as a "bony cage", and may bring better stability instantly and better fusion in the future; (5) removal of residual disc may help to achieve solid fusion; (6) the vertebral body osteotomy order was from inside to outside (eggshell technique) rather than from outside to inside, which means it is not necessary to expose the segmental vessels in most cases, with less vascular complications expected; and (7) VCD is a close-opening technique and the hinge of correction is adjustable, compared with the previous closing wedge osteotomy (CWO). The hinge in the CWO technique is positioned at the anterior longitudinal ligament at the apex of the deformity. It may become curved or kinked or potentially damaged if the spinal cord was shortened for a period too long during the correction. Theoretically, the more the hinge is posteriorly located, the smaller the need for shortening of the spinal cord, and thus safer correction.

In this study, most cases saw nearly normal restoration of the sagittal and coronal profiles. The average sagittal angle was corrected from a preoperative kyphosis of $100.3^{\circ}$ to a postoperative angle of $15.9^{\circ}$, with a mean correction of $84.1 \%$ in the kyphosis group. In the kyphoscoliosis group, the correction rate in the coronal plane was $64 \%$. At 2-year follow-up, all patients demonstrated a solid fusion at the osteotomy site and no pseudarthrosis was found.

We limited the indication of VCD to severe sharp angular spinal deformity, and the main consideration was to balance the manipulation itself, along with the potential achievement and complications. If a patient's spinal deformity was not severe $\left(<70^{\circ}\right.$ in the sagittal or coronal plane), one or two PSOs may be enough; if the height of the anterior column was near to normal vertebrae, e.g. in a round kyphosis, we also recommend an SPO or PSO. The VCD technique is a combination of several spinal surgery techniques, which is a technically demanding method; therefore, this procedure should be reserved for the most experienced spine surgeons.

\section{Conclusion}

For cases with sharp angular spinal deformity, VCD offers a safe and reliable way to achieve good results, including realignment of the deformed spine, decompression of the neurological elements, and potential improvement in neurologic function. In experienced hands, this can be achieved with an acceptable complication rate when compared with other procedures of similar magnitude.

\section{Key points}

1. The goal of management of sharp angular spinal deformity is to realign the spinal deformity and to decompress the neurological elements.

2. For cases with sharp angular spinal deformity, VCD offers a relatively safe and reliable way to achieve good results.

3. Residual bone of osteotomy site in VCD may take the place of metal mesh described in the VCR technique, which serves as a "bony cage" and may bring better stability instantly and better fusion in the future.

Conflict of interest No conflict of interest in the subject matter of this article. There was no funding source for this study.

Open Access This article is distributed under the terms of the Creative Commons Attribution Noncommercial License which permits any noncommercial use, distribution, and reproduction in any medium, provided the original author(s) and source are credited.

\section{References}

1. Boachie-Adjei O, Ferguson JI, Pigeon RG et al (2006) Transpedicular lumbar wedge resection osteotomy for fixed sagittal imbalance: surgical technique and farly results. Spine 31:485-492

2. Bradford DS, Tribus CB (1997) Vertebral column resection for the treatment of rigid coronal decompensation. Spine 22:1590-1599

3. Bridwell K (2006) Decision making regarding Smith-Petersen vs. pedicle subtraction osteotomy vs. vertebral column resection for spinal deformity. Spine 31:S171-S178

4. Bridwell K, Lewis S, Rinella A et al (2004) Pedicle subtraction osteotomy for the treatment of fixed sagittal imbalance. Surgical technique. J Bone Joint Surg Am 86:44-49

5. Ditunno JF Jr, Young W, Donovan WH et al (1994) The international standards booklet for neurological and functional classification of spinal cord injury. American Spinal Injury Association. Paraplegia 32:70-80

6. Gertzbein SD, Harris MB (1992) Wedge osteotomy for the correction of posttraumatic kyphosis. Spine 17:374-379 
7. Justin SS, Vincent YW, Christopher PA (2008) Vertebral column resection for rigid spinal deformity. Neurosurgery 63:A177A182

8. Kawaharu H, Tomita K (2005) Influence of acute shortening on the spinal cord: an experimental study. Spine 30:613-620

9. Kim YJ, Lenke LG, Bridwell KH et al (2004) Free hand pedicle screw placement in the thoracic spine: is it safe? Spine 29:333341

10. Lehmer SM, Keppler L, Buscup RS et al (1994) Posterior transvertebral osteotomy for adult thoracolumbar kyphosis. Spine 19:2060-2067

11. Lenke LG, Sides BA, Koester LA et al (2010) Vertebral column resection for the treatment of severe spinal deformity. Clin Orthop Relat Res 468:687-699

12. Lenke LG, O'Leary PT, Bridwell KH et al (2009) Posterior vertebral column resection for severe pediatric deformity: minimum two-year follow-up of thirty-five consecutive patients. Spine 34:2213-2221

13. Pappou IP, Papadopoulos EC, Swanson AN et al (2006) Pott disease in the thoracolumbar spine with marked kyphosis and progressive paraplegia necessitating posterior vertebral column resection and anterior reconstruction with a cage. Spine 31:E123E127

14. Suk SI, Chung ER, Lee SM et al (2005) Posterior vertebral column resection in fixed lumbosacral deformity. Spine 30:E703E710

15. Suk SI, Kim JH, Kim WJ et al (2002) Posterior vertebral column resection for severe spinal deformities. Spine 27:2374-2382

16. Thiranont N, Netrawichien P (1993) Transpedicular decancellation closed wedge vertebral osteotomy for treatment of fixed flexion deformity of spine in ankylosing spondylitis. Spine 18:2517-2522

17. Wang Y, Zhang Y, Zhang X et al (2008) A single posterior approach for multilevel modified vertebral column resection in adults with severe rigid congenital kyphoscoliosis: a retrospective study of 13 cases. Eur Spine J 17:361-372

18. Wang Y, Zhang Y, Zhang X et al (2009) Posterior-only multilevel modified vertebral column resection for extremely severe Pott's kyphotic deformity. Eur Spine J 18:1436-1442

19. White AA III, Panjabi MM (1990) Practical biomechanics of scoliosis and kyphosis: clinical biomechanics of the spine. 2nd ed. JB Lippincott, Philadelphia 127-68 